\title{
Vaccines, Virucides and Drugs Against HIV/AIDS: Hopes and Optimisms for the Future
}

\author{
A.A. Al-Jabri ${ }^{*}, 1$ and F.Q. Alenzi ${ }^{2}$ \\ ${ }^{I}$ Immunology Unit, Department of Microbiology and Immunology, College of Medicine and Health Sciences, Sultan \\ Qaboos University, Muscat, Oman \\ ${ }^{2}$ Department of Clinical Laboratory Sciences, College of Applied Medical Sciences, King Saud University, P.O. Box \\ 422, Alkaraj 11942, Saudi Arabia
}

\begin{abstract}
More than 25 million lives have been claimed by AIDS and 33.2 million people are estimated to have HIV, the majority of which are living in the underdeveloped countries. Failed tests on vaccines, virucides and complete virus eradication have caused scientists to refocus on the basic questions of what makes an effective HIV immune response. The "gloom" over disappointing research results on vaccine development and virucides "threatens to overshadow more positive" HIV/AIDS-related news, such as findings that male circumcision might reduce the likelihood of HIV transmission and that giving antiretroviral drugs to "high-risk" HIV-negative people (pre-exposure prophylaxis) could help protect them from infection. Something like pre-exposure prophylaxis has a good chance of becoming available before we have a $100 \%$ efficacious vaccine. The future in the field of HIV/AIDS will be much brighter if global research is appropriately coordinated and sufficient funds are available.
\end{abstract}

Keywords: HIV, AIDS, immune response, vaccines, virucides, drugs.

\section{INTRODUCTION}

To date, more than 25 million lives have been claimed by AIDS and 33.2 million people are estimated to have HIV, the majority of which are living in the underdeveloped countries. Failed tests on vaccines, microbicides and virus eradication have caused scientists to refocus on the basic questions of what makes an effective HIV immune response and how scientists can create neutralizing antibodies that block HIV infection [1]. Disappointing news in the field of HIV vaccine research came last year when Merck halted clinical trials of an experimental vaccine over safety concerns. The "gloom" over disappointing research results "threatens to overshadow more positive" HIV/AIDS-related news, such as findings that male circumcision might reduce the likelihood of HIV transmission and that giving antiretroviral drugs to "highrisk" HIV-negative people could help protect them from infection, a concept that is referred to as pre-exposure prophylaxis [2]. Something like pre-exposure prophylaxis has a good chance of becoming available before we have a $100 \%$ efficacious vaccine.

Since the beginning of the AIDS epidemic, scientists and clinicians were working with a disease that was not treatable at all, and the majority of patients died relatively quickly. In the nineties and due to better recognition of how HIV spreads and to new medications developed for treatments, the disease became more treatable. The next goal is a cure, in other words "complete viral eradication", having the ability

*Address correspondence to this author at the Department of Microbiology and Immunology, College of Medicine and Health Sciences, Sultan Qaboos University, P.O. Box 35, PC: 123, Muscat, Oman;

E-mail: aaljabri@squ.edu.om to remove the virus from the body or to suppress it to the point where it can no longer cause any damage. This goal is reachable as the progress we are making against HIV is much larger than in other comparable serious diseases. Currently medications are better than ever at treating AIDS patients and possibly even healing them.

In this article, we will discuss briefly the current situation with regard to vaccines, microbicides (virucides) and drugs against HIV/AIDS and the future expectation in these important areas.

\section{VACCINES}

Recently major vaccination studies have been stopped and we, unfortunately, do not expect to find a vaccine in the very near future [3]. However, there are other possibilities to prevent new infections. We know, for example, that people who do not have detectable viruses because they are successfully treated, hardly ever infect others. These individuals no longer pose a danger of infecting others as far as new infections are concerned. However, the AIDS pandemic would only be defeated by a preventative vaccine, rather than treating people who are already infected.

Out of the 50 candidates that have been evaluated among humans, only two vaccines have made it through all three phases of trials, and both were rejected as quite ineffective $[3,4]$. Most recently, one major vaccine trial was halted after early results showed that it appeared to place volunteers at greater risk of HIV infection $[3,4]$. Another vaccine in planning phases, involving tests among 8,500 individuals was found to be largely ineffective $[3,4]$. Despite these failures, about 30 vaccines remain in the pipeline, looking at different ways of stimulating the immune system $[3,4]$. 
Although HIV vaccine development is difficult, its benefits could be tremendous [5]. The challenges are huge, but with no doubt that we will live in a world without HIV some day. Vaccine researchers need to be unafraid to fail. We need to examine carefully why some vaccines have worked, and try and dissect why they have worked. More emphasis should be put on studying human immune responses and taking into consideration successful vaccines for other diseases such as influenza, yellow fever, and hepatitis-B as possibly useful models.

\section{VIRUCIDES}

Virucides are microbicidal agents that are able to destroy virus particles on contact. They differ from antivirals in that they are designed to act directly and rapidly by lysing viral membranes on contact $[6,7]$. Recently attention has been directed towards the possible use of these agents as a preventive factor against the sexual transmission of HIV infection. Sexual intercourse is an important route of HIV transmission and the need for an agent that can be used safely and effectively by women before sexual intercourse and without the need for agreement from the male partner demands more research from scientists. For example, the spermicidal nonoxynol-9, a non-ionic surfactant, possesses anti-HIV-1 activity and has been used experimentally as a means of reducing the risk of viral transmission [8]. However, this virucide has a damaging effect on the epithelial linings of the female genital tract, and is believed to enhance the susceptibility to HIV infection rather than preventing infection [7]. Therefore, new agents that possess virucidal activity without cytotoxicity on normal cells are required. Among many compounds being tested, bile salts with high surfactant activity may represent a class of compounds that can be used as virucides against HIV [6]. Their ability to inactivate high tittered HIV gives them the unique property of being used as biological disinfectants as well as virucide compounds.

Researchers' believed virucides delivered in a gel applicator, vaginal ring or capsule, could be a godsend to African women facing the threat of coercive sex by an infected partner. There have been nine fully-completed or halted trials of microbicide candidates, one of which showed that the prototype actually boosted the risk of infection by causing vaginal lesions that helped the virus to enter the bloodstream [8]. The new tack is to look at a gel that incorporates viruskilling drugs that are already tried-and-tested among people with HIV. At least five gels in this category are in the early stages of trials and despite past failures, there is a true cause for optimism in this field.

After the disappointing results from all vaccine efficacy trials conducted to date, the field of microbicides (virucides) research now faces many challenges. New promising strategies are now available. However, proper coordination between researchers need to be addressed to advance the next generations of candidates into clinical trials, and the use of appropriate animal models in this process will be critical [9].

\section{DRUGS}

The clinical course of AIDS was drastically changed in the nineties with the advent of highly active antiretroviral therapy (HAART). In the majority of AIDS patients, HAART leads to sustained reductions in HIV-1 replication, rise in CD4+ T-cell count, and concomitant immune reconstitution, resulting in significant reductions in morbidity and mortality. These benefits, however, have been achieved at the cost of considerable drug toxicity and monetary expense. It is well accepted that HAART alone will not cure HIV-1 infection because of the existence of persistent viral reservoirs, which ultimately rekindle viral replication once therapy is stopped [10]. Consequently, any attempts at viral eradication will depend on the implementation of therapeutic strategies to specifically target and clear these reservoirs. The best characterized reservoir to date is the pool of latently infected memory CD4+ T cells.

Using antiretroviral drugs as a "pre-exposure" prevention is another strategy being currently investigated. The idea is to have people at risk from infection take one drug, or a combination of more than one, before having sex to prevent infection. However, this may help the virus to mutate; in the same way that misusage of antibiotics can lead to drug resistance.

\section{THE FUTURE}

Despite the intensive research conducted to uncover the pathogenesis of HIV/AIDS, the whole picture of AIDS pathogenesis is still not clear. During HIV infection, there is a window period that we do not know much about. This is referred to as the window of vulnerability. This can become a window of opportunity because in that very short timeframe with the bursts of viremia and the establishment of a reservoir with a latent component occur. It is in that very small timeframe that our success or failure with vaccines as well with our ability to ultimately control, perhaps even cure HIV will rest. Virus reservoir is established literally within days of acute infection. Therefore, this window of vulnerability and opportunity is short. A recent paper showed that not only a reservoir is formed early, but byproducts of CD4 positive $\mathrm{T}$-cell deaths increase significantly within days and are capable of suppressing the human immune response to the virus [11]. So, we have a double whammy. We have a reservoir that almost immediately formed and we have products of the death of cells suppressing the immune response that would hopefully prevent the establishment of that reservoir. Therefore, future research should aim at reversing these processes.

Functional genomic screening, particularly large scale, small inhibitory RNA screening will in the future open up avenues of new targets. Selective small inhibitory RNAs, over 270 proteins were identified, only 36 of which have been previously implicated in HIV replication. There are scores and scores of intercellular targets that can be used in a new pipe line of interventions [12]. These anti-sense inhibitors hold promise as potential new agents to induce the latent reservoir without resulting in global cellular activation. However, anti-sense therapy is limited by its poor stability in the body and limited intracellular uptake and issues regarding in vivo delivery remain. As our understanding of the mechanisms controlling HIV-1 latency grows, additional agents will undoubtedly be discovered. 
There is an urgent need to broaden research and to answer fundamental questions in HIV/AIDS pathogenesis and vaccine development through laboratories, the use of nonhuman primate, and clinical research. In addition, it is important to attract and retain young researchers in the area of HIV pathogenesis, vaccine development and the discovery of new therapeutic agents.

\section{CONCLUSION}

Although results of HIV vaccine research have been "unrelentingly negative" in recent years, scientists should not give up and still should increase efforts to develop a vaccine. Big investments for the future are needed not only in the basic science of HIV prevention, but also in clinical trials for the search for an effective HIV vaccine. Collaboration for vaccine development, new virucides and new drugs need to be increased but avoiding wasted or duplicated efforts. Preexposure prophylaxis should be investigated in parallel with research for vaccines, virucides and new drugs. The future, in the field of HIV/AIDS, will be much brighter if global research is appropriately coordinated with appropriate funding being available.

\section{REFERENCES}

[1] Senior K. Back to basics for HIV vaccine research. Lancet Infect Dis 2008; 8: 467.
[2] Kersh EN, Luo W, Adams DR, et al. Short communication: no evidence of occult SHIV infection as demonstrated by CD8+ cell depletion after chemoprophylaxis-induced protection from mucosal infection in rhesus macaques. AIDS Res Hum Retroviruses 2008; 24: 543-6.

[3] Johnston MI, Fauci AS. An HIV vaccine--challenges and prospects. N Engl J Med 2008; 359: 888-90.

[4] Fauci AS, Johnston MI, Dieffenbach CW, et al. HIV vaccine research: the way forward. Science 2008; 321: 530-2.

[5] Al-Jabri AA. HIV/AIDS vaccines. How long must humanity wait? Sultan Qaboos Univ Med J 2007; 7: 193-5.

[6] Al-Jabri AA, Wigg MD, Elias E, Lambkin R, Mills CO, Oxford JS. In vitro anti-HIV-1 virucidal activity of tyrosine conjugated tri and dihydroxy bile salt derivatives. J Antimicrob Chemother 2000; 45: 617-21.

[7] Wilson DP, Coplan PM, Wainberg MA, Blower SM. The paradoxical effects of using antiretroviral-based microbicides to control HIV epidemics. Proc Natl Acad Sci USA 2008; 105: 9835-40.

[8] Food and Drug Administration, HHS. Over-the-counter vaginal contraceptive and spermicide drug products containing nonoxynol 9; required labeling. Final rule. Fed Regist 2007; 72: 71769-85.

[9] Grant RM, Hamer D, Hope T, et al. Whither or wither microbicides? Science 2008; 321: 532-4.

[10] Blankson J, Persaud D, Siliciano R. The challenge of viral reservoirs in HIV-1 infection. Annu Rev Med 2002; 53: 557-93.

[11] Haynes BF, Shattock RJ. Critical issues in mucosal immunity for HIV-1 vaccine development. J Allergy Clin Immunol 2008; 122: 39.

[12] Huan, J, Wang F, Argyris E, et al. Cellular microRNAs contribute to HIV-1 latency in resting primary CD4+ T lymphocytes. Nat Med 2007; 12: 1241-7. 